\title{
Note on paraconsistency and reasoning about fractions
}

\author{
Jan A. Bergstra and Inge Bethke** \\ Informatics Institute, University of Amsterdam \\ Science Park 904, 1098 XH Amsterdam, \\ The Netherlands
}

\begin{abstract}
We apply a paraconsistent strategy to reason about fractions.
\end{abstract}

Keywords: paraconsistent reasoning, mathematics education, fractions

\section{Introduction}

Suppose we want to define an arithmetic framework in which it is possible to reason about fractions in a consistent and reliable way and in which the usual laws of arithmetic hold: e.g. for natural numbers $n, l$ and $k \neq 0 \neq m$ the equations

$$
\frac{n}{m}+\frac{l}{k}=\frac{n k+l m}{m k} \quad \text { and } \quad \frac{n}{m}+\frac{l}{m}=\frac{n+l}{m}
$$

should be valid. At the same time, we want to consider fractions as mathematical expressions with typical syntactic operations like the numerator num( ) satisfying

$$
\operatorname{num}\left(\frac{n}{m}\right)=n .
$$

Then equational logic dictates

$$
n+l=\operatorname{num}\left(\frac{n+l}{m}\right)=\operatorname{num}\left(\frac{n}{m}+\frac{l}{m}\right)=\operatorname{num}\left(\frac{n m+l m}{m m}\right)=n m+l m .
$$

for arbitrary $l, n$ and $m \neq 0$, and our framework is inconsistent. Nevertheless, fractions are of great practical and abstract importance and we should be able to reason about them without lapsing into absurdity.

In mathematics education, one can get around this predicament in various ways: avoiding the concepts of numerator and denominator (see e.g. [8]), viewing fractions as heterogeneous subject (see e.g. 7]), or accepting cognitive conflicts (see e.g. 9]). In this note, we propose to apply paraconsistent reasoning.

**Corresponding author. Email:I.Bethke@uva.nl 
A paraconsistent logic is a way to reason about inconsistent information without exploding in the sense that if a contradiction is obtained, then everything can be obtained. Paraconsistent logics come in a broad spectrum, ranging from logics with the thought that if a contradiction were true, then everything would be true, to logics that claim that some contradictions really are true. In this note, we choose a particular paraconsistent reasoning strategy to tackle the dilemma sketched above. We do not claim that this is the only possible way how to proceed in our scenario; other paraconsistent approaches may be suitable as well.

\section{The $\mathrm{C} \& \mathrm{P}$ structure}

The approach taken here belongs to the preservationist school. The fundamental idea is that, given an inconsistent collection of premises, one should not try to reason about the collection of premises as a whole, but rather focus on internally consistent subsets of premises. In 2004, Brown and Priest 4 introduced the Chunk and Permeate (C \& P) strategy for dealing with reasoning situations involving incompatible assumptions. In this reasoning strategy, a theory is broken up into chunks and only restricted information is allowed to pass from one chunk to another. In what follows we give a precise rendering of this idea.

Let $L$ be some classical language and let $\vdash$ be an appropriate classical consequence relation. If $\Gamma$ is a set of sentences in $L, \Gamma^{\vdash}$ denotes the closure of $\Gamma$ under $\vdash$. A covering of $\Gamma$ is a set $\left\{\Gamma_{i} \mid i \in I\right\}$ of classical consistent sets of sentences such that $\Gamma=\bigcup_{i \in I} \Gamma_{i}$. If $C=\left\{\Gamma_{i} \mid i \in I\right\}$ is a covering of $\Gamma$, a permeability relation $\rho$ on $C$ is a map from $I \times I$ to sets of sentences of $L$. If $i_{0} \in I,\left\langle C, \rho, i_{0}\right\rangle$ is called a $C \& \& P$ structure on $\Gamma$.

If $\mathcal{P}=\left\langle C, \rho, i_{0}\right\rangle$ is a $\mathrm{C} \& \mathrm{P}$ structure on $\Gamma$ and $A$ is a sentence in the language under consideration, then

$$
\Gamma \vdash_{\mathcal{P}} A \text { if and only if } A \in \Gamma_{i_{0}}^{\omega}
$$

where

$$
\begin{aligned}
\Gamma_{i}^{0} & =\Gamma_{i}^{\vdash} \\
\Gamma_{i}^{n+1} & =\left(\Gamma_{i}^{n} \cup \bigcup_{j \in I}\left(\Gamma_{j}^{n} \cap \rho(j, i)\right)\right)^{\vdash} .
\end{aligned}
$$

The $\mathrm{C} \& \mathrm{P}$ consequences of $\Gamma$ are thus sentences that can be inferred in the designated chunk $i_{0}$ when information has been allowed to flow from other chunks along the permeability relation.

In our case, a simple binary C \& $\mathrm{P}$ structure will be sufficient. We partition our inconsistent theory into two chunks: the source chunk $\Gamma_{S}$ and the target chunk $\Gamma_{T}$. The flow of information is from the former to the latter only and the target chunk is the output chunk. Note that in this case, $\Gamma_{S}^{\omega}=\Gamma_{S}^{\vdash}$ and hence

$$
\Gamma_{T}^{\omega}=\Gamma_{T}^{1}=\left(\Gamma_{T} \cup\left(\Gamma_{S}^{\vdash} \cap \rho(S, T)\right)\right)^{\vdash} .
$$

We let $L$ be the language of equational logic with terms over the two-sorted signature $\Sigma$ containing 0,1 , the numerator and denominator num and denom, 


$$
\begin{aligned}
n+0 & =n \\
(n+m)+l & =n+(m+l) \\
n+m & =m+n \\
n \cdot 1 & =n \\
(n \cdot m) \cdot l & =n \cdot(m \cdot l) \\
n \cdot m & =m \cdot n \\
n \cdot(m+l) & =n \cdot m+n \cdot l \\
\frac{n}{m} \cdot \frac{l}{k} & =\frac{n \cdot l}{m \cdot k} \\
\frac{n}{m}+\frac{l}{k} & =\frac{n \cdot k+l \cdot m}{m \cdot k} \\
\frac{n}{m}+\frac{l}{m} & =\frac{n+l}{m} \\
m \neq 0 \neq k \rightarrow \quad \alpha+\beta)+\gamma & =\alpha+(\beta+\gamma) \\
\alpha+\beta & =\beta+\alpha \\
m \neq 0 \rightarrow \beta) \cdot \gamma & =\alpha \cdot(\beta \cdot \gamma) \\
\alpha \cdot \beta & =\beta \cdot \alpha \\
\alpha \cdot(\beta+\gamma) & =\alpha \cdot \beta+\alpha \cdot \gamma \\
n \text { num }\left(\frac{n}{m}\right) & =n \\
\text { denom }\left(\frac{n}{m}\right) & =m \\
m \neq 0 \wedge \text { a } &
\end{aligned}
$$

Table 1: The inconsistent equational theory $\Gamma$

the fraction -, symbols for addition and multiplication of natural numbers and fractions, respectively, and an additional error element $a$ produced by division by zero. The inconsistent theory $\Gamma$ is the equational theory of the set of axioms given in Table 1. Here $k, l, m, n$ range over natural numbers, and $\alpha, \beta, \gamma$ denote fractions. The binary $\mathrm{C} \& \mathrm{P}$ structure for our problem is $\left\langle\left\{\Gamma_{S}, \Gamma_{T}\right\}, \rho, T\right\rangle$ where

- the source chunk $\Gamma_{S}$ is the theory of the axioms (1) - (8), (11) - (17) of Table 1 and the axioms (18) and (19) of Table 2

- the target chunk $\Gamma_{T}$ consists of the axiom (20) of Table 2, and

- the information $\rho$ that is allowed to flow from source to target consists of the axioms of the source except for (16) and (17).

Observe that in the source, fractions with identical denominators can be 


$$
\begin{aligned}
m \neq 0 \rightarrow & \frac{n}{m}=\frac{n}{1} \cdot \frac{1}{m} \\
\frac{n}{1}+\frac{m}{1} & =\frac{n+m}{1} \\
m \neq 0 \neq k \rightarrow \quad \frac{n \cdot k}{m \cdot k} & =\frac{n}{m}
\end{aligned}
$$

Table 2: Alternative axioms for source and target

added $(\dagger)$ : for $m \neq 0$ we have

$$
\begin{aligned}
\frac{n}{m}+\frac{k}{m} & =\frac{n}{1} \cdot \frac{1}{m}+\frac{k}{1} \cdot \frac{1}{m} \\
& =\left(\frac{n}{1}+\frac{k}{1}\right) \cdot \frac{1}{m} \\
& =\frac{n+k}{1} \cdot \frac{1}{m} \\
& =\frac{n+k}{m}
\end{aligned}
$$

This fact together with axiom (20) yields axiom (9). Thus $\left\{\Gamma_{S}, \Gamma_{T}\right\}$ covers $\Gamma$.

That $\Gamma_{S}$ is consistent can be seen as follows. We let $M$ be the $\Sigma$-algebra with the sorts $\mathbb{N}_{a}=\mathbb{N} \cup\{a\}$ and $\mathbb{F}=\mathbb{N} \times \mathbb{N}$ where the operations are interpreted as follows. If $\circ \in\{+, \cdot\}$, then $\circ: \mathbb{N}_{a} \times \mathbb{N}_{a} \rightarrow \mathbb{N}_{a}$ is defined by

$$
x \circ y= \begin{cases}x \circ y & \text { if } x, y \in \mathbb{N} \\ a & \text { otherwise }\end{cases}
$$

and $-: \mathbb{N}_{a} \times \mathbb{N}_{a} \rightarrow \mathbb{F}$ is defined by

$$
\frac{x}{y}= \begin{cases}(x, y) & \text { if } x \in \mathbb{N}, y \in \mathbb{N} \\ (0,0) & \text { otherwise }\end{cases}
$$

num, denom $: \mathbb{F} \rightarrow \mathbb{N}_{a}$ are defined by

$$
\operatorname{num}((n, m))= \begin{cases}n & \text { if } m \neq 0 \\ a & \text { otherwise }\end{cases}
$$

and

$$
\operatorname{denom}((n, m))= \begin{cases}m & \text { if } m \neq 0 \\ a & \text { otherwise }\end{cases}
$$


and $+, \cdot: \mathbb{F} \times \mathbb{E} \rightarrow \mathbb{F}$ are defined by

$$
(n, m)+(l, k)= \begin{cases}(n+l, m) & \text { if } m=k \neq 0 \\ (0,0) & \text { otherwise }\end{cases}
$$

and

$$
(n, m) \cdot(l, k)= \begin{cases}(n l, m k) & \text { if } m \neq 0 \neq k \\ (0,0) & \text { otherwise }\end{cases}
$$

Note that $M$ does not satisfy the full axiom of fraction addition (9). It is easy to see that $M$ is a model for $\Gamma_{S}$. Clearly, $\Gamma_{T}$ is consistent. $\Gamma_{T} \cup \rho$ is consistent too: $\mathbb{Q}_{0}$ and $\mathbb{Q}_{a}$ - the zero-totalized and the $a$-totalized meadow of the rational numbers (see e.g. [1, 3]), respectively - are both models of $\Gamma_{T} \cup \rho$. We have thus arrived at a consistent theory where we have full addition of fractions: for $m \neq 0 \neq l$

$$
\begin{aligned}
\frac{n}{m}+\frac{k}{l} & =\frac{n l}{m l}+\frac{k m}{l m} \\
& =\frac{n l}{m l}+\frac{k m}{m l} \\
& =\frac{n l+k m}{m l}
\end{aligned}
$$

\section{Conclusion}

$M$ is only one of a number of possible structures that incorporate fractions in such a manner that the oprations numerator and denominator can be properly defined. We do not exclude that more convincing datatypes can be found as models for $\Gamma_{S}$. For instance with / representing integer division, the defining equation

$$
\frac{n}{m}+\frac{k}{l}=\frac{(n \cdot l+k \cdot m) / \operatorname{gcd}(m, l)}{m \cdot l / \operatorname{gcd}(m, l)}
$$

is compatible with having functions num and denom around in combination with associativity and the rule

$$
\frac{n}{m}+\frac{k}{m}=\frac{n+k}{m}
$$

We will not explore such alternatives in this note. For meadows different from common meadows see e.g. [2].

The relation between the views $\Gamma_{S}$ and $\Gamma_{T}$ requires further attention. Following the original line of thought, $\Gamma_{S}$ is a stepping stone towards $\Gamma_{T}$ which in our case implies that the application of the $\mathrm{C} \& \mathrm{P}$ strategy does not lead to a picture where fractions and rationals coexist with the operations num and denom.

It seems plausible to imagine that a person thinking along these lines about fractions switches back and forth between $\Gamma_{S}$ and $\Gamma_{T}$. The situation may then be 
compared with how a human observer looks at the Necker cube [5, 6]. This cube is a 2-dimensional figure which invites an observer to "see" in an alternating manner two different 3-dimensional interpretations of it. Instead of the 3rd dimension we consider a logic, informally understood. And $\Gamma_{S}$ and $\Gamma_{T}$ represent two logics of fractions the alternation of which seems to be adequately described by the inconsistency-tolerant technique of $\mathrm{C} \& \mathrm{P}$.

\section{References}

[1] Bergstra, J.A., Bethke, I., and Ponse, A. (2013). Cancellation meadows: a generic basis theorem and some applications. The Computer Journal, 56(1):3-14, doi:10.1093/comjnl/bsx147. (Also available at arXiv:0803.3969 [math.RA, cs.LO].)

[2] Bergstra, J.A. and Middelburg, C.A. (2015). Division by zero in non-involutive meadows . Journal of Applied Logic, 13(1):1-12, doi:10.1016/j.jal.2014.10.001. (Also available at arXiv:1406.2092 [math.RA, cs.LO].)

[3] Bergstra, J.A. and Ponse, A. (2015). Division by zero in common meadows. In de Nicola, R. and Hennicker, R. (eds.), Software Services and Systems, LNCS 8950, pp. 46-61. Springer. (Also available at arXiv:1406.3280v2 [cs.LO].)

[4] Brown, B. and Priest, G. (2004). Chunk and Permeate, a paraconsistent inference strategy. Part I: the infinitesimal calculus. Journal of Philosophical Logic, 33:379 -388.

[5] Mortensen, Ch. (2006). An Analysis of Inconsistent and Incomplete Necker Cubes. Australasian Journal of Logic, 4:216-225.

[6] Necker, L.A. (1832). Observations on some remarkable optical phaenomena seen in Switzerland; and on an optical phaenomenon which occurs on viewing a figure of a crystal or geometrical solid. London and Edinburgh Philosophical Magazine and Journal of Science, 1(5):329-337.

[7] Padberg, F. (2012). Didaktik der Bruchrechnung (4th edition). Series: Mathematik, Primar- und Sekundarstufe, Springer-Spektrum.

[8] Rollnik, S. (2009). Das pragmatische Konzept für den Bruchrechenunterricht. $\mathrm{PhD}$ thesis, University of Flensburg, Germany.

[9] Tall, D. and Vinner, S. (1981). Concept image and concept definition in mathematics, with special reference to limits and continuity. Educational Studies in Mathematics, 12 151-169. 